\section{Sterbehilfe in der Schweiz}

M. Marti*

\section{Einleitung}

Seit längerer Zeit wird die Frage der Zulässigkeit von Sterbehilfemassnahmen kontrovers diskutiert; dies nicht nur in medizinischen, sondern auch in religiösen, philosophischen, aber auch juristischen Kreisen. Die Diskussion ist komplex, da sie in entscheidender Art und Weise auch auf moralische und ethische Standpunkte Rücksicht zu nehmen hat.

Nachdem während langer Zeit die Zulässigkeit von Massnahmen im Bereich der Sterbehilfe durch eine christlich geprägte Wertordnung unterdrückt wurde, fanden in jüngerer Zeit vermehrt Tendenzen Beachtung, welche einer Tabuisierung entgegenwirken und derartigen Massnahmen - in einem mehr oder weniger engen Korsett - offener gegenüberstehen. Dies führte mitunter dazu, dass in etlichen Staaten der Versuch unternommen wurde, regulatorische Leitplanken zu setzen. Als Beispiel für eine liberale Praxis wird dabei immer wieder Holland genannt. Die Rechtsprechung in Holland sieht vor, dass die (direkte) aktive Sterbehilfe in gewissen Fällen keine strafrechtlichen Konsequenzen für den Arzt nach sich zieht, da sich dieser in einer die Tat rechtfertigenden Notstandssituation befindet.

Auch in der Schweiz ist die politische Diskussion lanciert worden. Im Jahr 1994 reichte Nationalrat Ruffy eine Motion ein, in welcher er eine neue Regelung der Sterbehilfe im Strafgesetzbuch forderte.

Der folgende Beitrag soll zeigen, welche gesetzlichen Bestimmungen heute im Bereich der Sterbehilfe und der Beihilfe zum Suizid zu beachten sind, und gibt einen Überblick über die laufenden Reformbemühungen. Hingegen wird vorliegend bewusst auf eine Wertung und Stellungnahme verzichtet. Die sich stellenden Fragen eignen sich weder für polemische Debatten noch für werberisches Wirken, sondern bedürfen der ehrlichen und persönlichen Auseinandersetzung eines jeden Betroffenen.

\footnotetext{
* Der Autor ist Rechtsanwalt bei Kellerhals \& Partner, Bern, sowie wissenschaftlicher Assistent an der Universität Bern. Er ist zudem Dozent an der Privaten Hochschule Wirtschaft und unterrichtet Recht in den Kursen Medical Manager FH und VSAO Management Curriculum.
}

Korrespondenz:

lic. iur. Mario M. Marti, Fürsprecher

Kellerhals \& Partner

Kapellenstrasse 14

CH-3011 Bern

\section{Gesetzliche Grundlagen}

Keine positivrechtliche Regelung

Im heute geltenden Schweizerischen Recht besteht keine ausdrückliche gesetzliche Regelung der Sterbehilfe: weder existiert ein Gesetz, in welchem die Sterbehilfe ausdrücklich verboten oder zugelassen wäre, noch ein solches, in welchem diesbezügliche Voraussetzungen und Verfahren genannt wären. Vielmehr wird die Zulässigkeit der Sterbehilfe im Rahmen der strafrechtlichen Würdigung des Todesfalles geprüft.

\section{Strafgesetzbuch}

\section{Vorsätzliche Tötung (Art. 111 StGB ${ }^{1}$ )}

Tatbestand. Das Schweizerische Strafrecht geht von einem grundsätzlichen Tötungsverbot aus. Nach Art. 111 StGB macht sich strafbar, wer einen Menschen vorsätzlich tötet, unabhängig von weiteren Voraussetzungen, namentlich davon, in welcher Situation sich die getötete Person befand.

Aktive Sterbehilfe. Unter aktiver Sterbehilfe wird die gezielte Tötung eines schwerkranken, leidenden $\mathrm{Pa}-$ tienten durch einen Arzt oder eine dritte Person verstanden. Derartige Handlungen erfüllen ohne weiteres den Tatbestand der vorsätzlichen Tötung [1, 2]. Hehre Motive des Täters bzw. eine ausweglose Situation auf seiten des Patienten vermögen an der Strafbarkeit der Handlung nichts zu ändern, ebensowenig der Wille des Patienten [3]. Ein übergeordneter Rechtfertigungsgrund besteht nicht.

Die Vornahme einer Handlung im Sinne einer aktiven Sterbehilfe führt somit zwingend zur Bestrafung des Sterbehelfers.

Indirekt aktive Sterbehilfe. Durch die Verabreichung schmerzmildernder Medikamente an einen todkranken Patienten kann als Nebenfolge der Eintritt des Todes begünstigt oder beschleunigt werden. Sind solche Nebenfolgen bekannt und weiss der verabreichende Arzt um diese Wirkung, so handelt er in Bezug auf die Tötung des Patienten eventualvorsätzlich, d.h. er nimmt den Tod des Patienten in Kauf.

Obschon auch in dieser Konstellation der Tatbestand von Art. 111 StGB erfüllt wäre, verzichtet die juristische Lehre und Praxis auf eine Ahndung derartiger Delikte. Es wird argumentiert, dass die Bewahrung des Patienten vor unerträglichen Schmerzen zur gewohnheitsrechtlichen Berufspflicht des Arztes gehöre [3] und somit der Rechtfertigungsgrund von Art. 32 StGB vorliege (Rechtfertigung einer Tat, deren Begehung von einer Berufspflicht verlangt wird) [2]. Die Zulässigkeit der indirekt aktiven Sterbehilfe kann auch aus dem (privatrechtlichen) Behandlungsvertrag zwischen Arzt und Patient abgeleitet werden: Der Behandlungsvertrag erteilt dem Arzt nicht nur einen Heilauftrag, sondern verpflichtet ihn auch, zur Linderung der Leiden des Patienten beizutragen [2, 4].

1 Schweizerisches Strafgesetzbuch vom 21.12.1937 (StGB; SR 311.0). 
Ein derartiger Medikamenteneinsatz gilt als zulässig, sofern er im Hinblick auf die Schmerzmilderung notwendig ist $[1,3,5]$.

Passive Sterbehilfe

\section{- Tötung durch Unterlassung}

Bei der passiven Sterbehilfe erfolgt die Tötung einer Person nicht durch eine aktive Handlung, sondern durch eine Unterlassung, namentlich durch die Nichtausführung einer lebensverlängernden Behandlung. Unterlassungsdelikte sind nur strafbar, wenn der Täter gegenüber dem Opfer eine Garantenstellung innehatte, d.h. wenn ihm eine (gesetzliche oder vertragliche) Handlungspflicht obliegt. ${ }^{2}$ Dem behandelnden Arzt kommt regelmässig eine solche Garantenstellung aufgrund des Behandlungsvertrages zu [3]. Grundsätzlich ist demnach die Tötung durch Unterlassung als Tötungsdelikt strafbar.

- Selbstbestimmungsrecht des Patienten Die Garantenstellung des Arztes, d.h. seine Pflicht, den Patienten umfassend zu behandeln, steht aber in einem Spannungsverhältnis zum Selbstbestimmungsrecht des Patienten. Das Selbstbestimmungsrecht gibt dem Patienten die alleinige Macht, über seinen Körper zu entscheiden. ${ }^{3}$ Verzichtet er auf einen lebensnotwendigen Eingriff, ist dieser Entscheid zu respektieren; dem Arzt steht kein Recht auf Behandlung zu. Jeder Eingriff, der gegen den Willen des Patienten vorgenommen wird, ist infolge fehlender Einwilligung des Patienten ohne weiteres widerrechtlich. Dies bedeutet, dass in solchen Fällen der Arzt, der eine notwendige Behandlung unterlässt, nicht strafbar ist. Der Verzicht auf lebenserhaltende Massnahmen kann indessen nur vom urteilsfähigen $\mathrm{Pa}$ tienten gültig getroffen werden.

- Urteilsfähiger Patient

Der urteilsfähige Patient, also ein Patient, der in der Lage ist, vernunftgemäss zu handeln (Art. 16 ZGB), kann frei über seine Behandlung entscheiden. Der Entscheid ist vom Arzt zu respektieren. Damit der Patient über sein Schicksal frei entscheiden kann, muss er vorgängig vom Arzt über die Folgen und Risiken des Entscheides umfassend und unter Berücksichtigung seiner aktuellen Situation und Bedürfnisse aufgeklärt werden. ${ }^{4}$

- Urteilsunfähiger Patient

Ein urteilsunfähiger Patient ist nicht in der Lage, rechtsverbindliche Handlungen vorzunehmen (Art. 18 ZGB). Er kann somit auch nicht gültig auf eine lebenserhaltende Massnahme verzichten; Äusserungen eines urteilsunfähigen Patienten sind für den Arzt nicht bindend.

Für den unmündigen Sterbenden entscheidet in erster Linie dessen gesetzlicher Vertreter (Eltern, Vormund). Gegen dessen Willen dürfen lebenserhaltende Massnahmen weder abgebrochen noch verweigert werden. ${ }^{5}$ Auf der anderen Seite hat sich der Entscheid des gesetzlichen Vertreters zugunsten eines Verzichts auf lebenserhaltende Massnahmen am Wohle des Kindes bzw. des Be- vormundeten zu orientieren. Weigerungen des gesetzlichen Vertreters zur Vornahme von medizinischen Handlungen, die missbräuchlich sind und offensichtlich nicht im Interesse des urteilsunfähigen Patienten liegen, können vom behandelnden Ärzteteam übergangen werden [7].

In den Fällen, in welchen kein gesetzlicher Vertreter bestimmt ist, liegt der Entscheid über die Durchführung oder den Abbruch der Behandlung beim Arzt, wobei dieser sich am mutmasslichen Willen des Patienten zu orientieren hat [3]. Der mutmassliche Wille des Patienten ist aufgrund der gesamten Lebensumstände des Sterbenden zu ermitteln, hierzu sind regelmässig Gespräche mit nahen Angehörigen zu führen. Eine Patientenverfügung, in welcher der Patient im noch urteilsfähigen Zustand den Entscheid vorweggenommen hat, ist für den Arzt grundsätzlich nicht verbindlich. Sie stellt indessen eine starke Vermutung [8] für den mutmasslichen Willen des Patienten dar und wird wohl nur in Ausnahmefällen vom Arzt missachtet werden können.

\section{Tötung auf Verlangen (Art. 114 StGB)}

Am oben Gesagten ändert sich grundsätzlich auch dann nichts, wenn der Patient die Tötung vom Arzt oder Sterbehelfer ausdrücklich verlangt hat. Zwar kommt dann nicht mehr Art. 111 StGB als Grundtatbestand zur Anwendung, sondern der privilegierte Tatbestand von Art. 114 StGB. Auch in diesem Fall Tötung auf Verlangen aus achtenswerten Beweggründen - macht sich ein Sterbehelfer indessen grundsätzlich strafbar.

Der Wille des Patienten und die hehren Motive der Tötung haben somit keinen Einfluss auf die grundsätzliche Strafbarkeit der Sterbehilfe.

\section{Beihilfe zum Selbstmord (Art. 115 StGB)}

Das Selbstbestimmungsrecht des Patienten hat im Strafrecht die Straflosigkeit des Selbstmordversuchs zur Konsequenz. Dagegen stellt das Gesetz die Beihilfe zum Selbstmord unter Strafe, sofern sie aus selbstsüchtigen Gründen, d.h. zur Erlangung eines persönlichen Vorteils für den Helfer, erfolgte. Liegen keine solchen selbstsüchtigen Motive vor, ist die Beihilfe zum Selbstmord grundsätzlich zulässig.

Die Beurteilung, ob das Verhalten eines Arztes oder eines Dritten, der am Tod eines Patienten beteiligt war, strafrechtlich unter dem Aspekt der vorsätz-

\footnotetext{
${ }^{2}$ Weiter setzt die Strafbarkeit eines Unterlassungsdelikts die Tatmacht des Täters sowie die Tatsache, dass die Verletzung des Rechtsguts (hier: Leben) bei Vornahme der gebotenen Massnahme ausgeblieben wäre, voraus.

${ }^{3}$ Dies ergibt sich aus Art. 10 der Bundesverfassung (BV; SR 101) und Art. 28 des Zivilgesetzbuchs (ZGB; SR 210).

${ }^{4}$ Zur Frage der Aufklärung vgl. z.B. [6] sowie Art. 10 der Standesordnung FMH vom 12.12.1996.

${ }^{5}$ Vgl. Ziff. II., 3.3 der "Richtlinie für die ärztliche Betreuung sterbender und zerebral schwerst geschädigter Patienten" der Schweizerischen Akademie der Medizinischen Wissenschaften.
} 
lichen Tötung oder der Beihilfe zum Selbstmord zu beurteilen ist, hängt entscheidend von der Frage ab, ob dem Sterbewilligen bezüglich seines eigenen Todes die Tatherrschaft zustand oder nicht [5]. Hat der Sterbewillige die Macht über die Ausübung der Tötung, etwa indem er selber das todbringende Medikament zu sich nimmt, begeht er Selbstmord; ist die finale Handlung hingegen dem Sterbehelfer zuzuordnen, liegt kein Selbstmord vor, vielmehr sind die Regeln der aktiven oder passiven Sterbehilfe anzuwenden.

Richtlinien der Schweizerischen Akademie der Medizinischen Wissenschaften

Die Akademie der Medizinischen Wissenschaften hat medizinisch-ethische Richtlinien für die ärztliche Betreuung sterbender und zerebral schwerst geschädigter Patienten erlassen. ${ }^{6}$

Die Richtlinien umschreiben die Voraussetzungen, unter denen die passive und die indirekt aktive Sterbehilfe zulässig sind (Ziff. II. 1.2, 1.3, 2 und 3). Die aktive Sterbehilfe wird ausdrücklich verboten (Ziff. II. 1.4). Die Beihilfe zum Suizid wird als «kein Teil der ärztlichen Tätigkeit» bezeichnet (Ziff. II. 2.2).

Die Richtlinien der Akademie der Medizinischen Wissenschaften sind integraler Bestandteil des FMHStandesrechts. Es handelt sich hierbei grundsätzlich nicht um staatliches Recht, sondern um privates (Vereins-)Recht. Somit hat die Verletzung der Standesregeln auch keine staatlichen Sanktionen zur Folge; vielmehr können standesrechtliche Massnahmen verfügt werden. Ein Strafrichter wird dagegen die rechtliche Beurteilung der Sterbehilfe alleine aufgrund der einschlägigen strafrechtlichen Bestimmungen vornehmen.

\section{Laufende Revisionsbemühungen}

\section{Arbeitsgruppe Sterbehilfe}

1994 reichte Nationalrat Victor Ruffy im Parlament eine Motion ein, welche eine Lockerung der heutigen Strafbestimmungen forderte. 1996 wurde dieser Vorstoss als Postulat an den Bundesrat überwiesen, welcher darauf eine Arbeitsgruppe einsetzte. Diese erarbeitete in der Folge Vorschläge für eine gesetzliche (Neu)regelung. ${ }^{7}$

Die Arbeitsgruppe ist einstimmig der Meinung, dass die passive und die indirekt aktive Sterbehilfe im StGB gesetzlich geregelt werden sollten, verzichtet aber auf einen ausformulierten Vorschlag. Vertreten wird folgende Grundhaltung:

\section{Passive Sterbehilfe}

Wer aufgrund der Bitte eines Patienten auf lebensverlängernde Massnahmen verzichtet, macht sich nicht strafbar, dies unabhängig vom Zustand des Patienten oder der medizinischen Prognose.

\section{Indirekte aktive Sterbehilfe}

Bezüglich der indirekt aktiven Sterbehilfe befürwortet die Arbeitsgruppe eine weite Formulierung, so dass palliative Massnahmen nicht in Frage gestellt werden. Der Rechtfertigungsgrund soll nicht nur erfüllt sein, wenn der Arzt die Verkürzung des Lebens als Nebenfolge für möglich hält, sondern auch wenn er diese als so gut wie sicher anschaut.

In der Frage der Zulässigkeit der aktiven Sterbehilfe ist die Arbeitsgruppe geteilter Meinung. Eine knappe Mehrheit ist der Ansicht, Art. 114 StGB sei durch einen neuen Abs. 2 zu ergänzen, welcher vorsieht, dass der Täter in extremen Ausnahmefällen (unheilbare Beeinträchtigung der Gesundheit des Patienten, Erlösung von unerträglichen und nicht behebbaren Leiden) von der Strafe befreit wird (Strafbefreiungsklausel).

\section{Bericht des Bundesrates}

Der Bundesrat nahm zum Bericht der Arbeitsgruppe Stellung. ${ }^{8}$ Er sprach sich dabei aus für:

- eine Förderung der palliativen Medizin,

- eine ausdrückliche Regelung der passiven und indirekt aktiven Sterbehilfe (im StGB oder einem Spezialerlass).

Hingegen lehnt der Bundesrat aus grundsätzlichen Erwägungen ("Tabubruch») die Lockerung des Tötungsverbotes im Sinne einer ausnahmsweisen $\mathrm{Zu}$ lassung der direkten aktiven Sterbehilfe (gem. dem Vorschlag der Mehrheit der Arbeitsgruppe) ab.

\section{Ausblick}

Die Diskussion zur Sterbehilfe und zur Beihilfe zum Suizid ist noch nicht abgeschlossen. Die politische Auseinandersetzung hat bereits begonnen und wird weitergeführt: Im Parlament wurden verschiedene Vorstösse eingereicht: Die Parlamentarische Initiative Cavalli [9] verlangte die Regelung der aktiven Sterbehilfe, wie sie von der Mehrheit der Expertenkommission gefordert wird, d.h. im Sinne einer Lockerung der absoluten Strafbarkeit. Die Parlamentarische Initiative Vallender [10] verlangte eine Neufassung der Bestimmung über die Beihilfe zum Selbstmord, namentlich sollte nicht nur strafbar sein, wer aus selbstsüchtigen Beweggründen Beihilfe zum Suizid leistet, sondern grundsätzlich auch, wer - ohne Rücksicht auf die Beweggründe - einer nicht urteilsfähigen Person Beihilfe leistet oder wer dies gegenüber einer urteilsfähigen Person tut, ohne dabei aus dem persönlichen Umfeld der sterbewilligen Person zu stammen (sondern z. B. als Arzt handelt). Beide Initiativen wurden vom Nationalrat am 11. Dezember 2001 abgelehnt. ${ }^{9}$ Hingegen überwies der Nationalrat gleichen-

\footnotetext{
${ }^{6}$ Die Richtlinien sind abrufbar unter www.samw.ch/content/Richtlinien/d_Sterbehilfe.pdf.

${ }^{7}$ Der Bericht der Arbeitsgruppe findet sich unter www.ofj.admin.ch/themen/stgb-sterbehilfe/b-bericht-d.pdf.

${ }^{8}$ Der Bericht des Bundesrates findet sich unter www.ofj.admin.ch/themen/stgb-sterbehilfe/ber-ruffy-d.pdf.

${ }^{9}$ Die Kommission für Rechtsfragen des Nationalrates hat die Initiative Cavalli zur Annahme, diejenige von Vallender zur Ablehnung empfohlen.
} 
tags mit deutlicher Mehrheit eine erst jüngst eingereichte Motion Zäch [11], welche die gesetzliche Regelung der indirekt aktiven und der passiven Sterbehilfe sowie ein Massnahmepaket zur speziellen Förderung der Palliativmedizin fordert. Der Bundesrat ist somit beauftragt, dem Parlament eine entsprechende Gesetzesänderung vorzulegen. Bundesrätin Metzler sprach sich im Nationalrat für ein «behutsames Vorgehen" aus und erwartet "langwierige Arbeiten".

Grundsätzlich ist davon auszugehen, dass auch nach dem Entscheid des Nationalrates vom 11. Dezember 2001 die Debatte weitergehen wird. Kontrovers wird dabei weiterhin primär die Frage sein, ob im Bereich der aktiven Sterbehilfe eine Lockerung der Strafbestimmungen vorgenommen werden soll. Für die einen würde diese Lockerung modernen Bedürfnissen entsprechen, für die anderen käme dies einem eigentlichen Tabubruch gleich.

Mehr Einigkeit dürfte bezüglich der passiven und der indirekt aktiven Sterbehilfe bestehen. Die nun auszuarbeitende gesetzliche Regelung wird allen Betroffenen dienen und zu grösserer Rechtssicherheit führen.

\section{Literatur}

1 Stratenwerth G. Schweizerisches Strafrecht. Besonderer Teil I. Bern: Stämpfli; 1995.

2 Giger H. Reflexionen über Tod und Recht. Zürich: Orell Füssli; 2000.

3 Rehberg S. Arzt und Strafrecht. In: Honsell H. Handbuch des Arztrechts. Zürich: Schulthess; 1994.

4 Bericht der Arbeitsgruppe Sterbehilfe (s. Fussnote 7).

5 Trechsel S. Schweizerisches Strafgesetzbuch. Kurzkommentar. 2. Auflage. Zürich: Schulthess; 1997.

6 Wiegand W. Die Aufklärungspflicht und die Folgen ihrer Verletzung. In: Honsell H. (Hrsg.). Handbuch des Arztrechts. Zürich: Schulthess; 1994.

7 Bucher A. Natürliche Personen und Persönlichkeitsschutz. 3. Auflage. Basel: Helbing \&t Lichtenhahn; 1999.

8 Reusser K. Patientenwille und Sterbebeistand. Zürich: Schulthess; 1994.

9 Cavalli F. Parlamentarische Initiative «Strafbarkeit der aktiven Sterbehilfe. Neuregelung» (00.441). Eingereicht im Nationalrat am 27.9.2000.

10 Vallender D. Parlamentarische Initiative "Verleitung und Beihilfe zur Selbsttötung. Neufassung von Artikel 115 StGB» (01.407). Eingereicht im Nationalrat am 14.3.2001.

11 Zäch G. Motion «Sterbehilfe. Gesetzeslücke schliessen statt Tötung erlauben» (01.3523). Eingereicht im Nationalrat am 3.10.2001. 